\title{
A convenient band-gap interpolation technique and an improved band line-up model for InGaAlAs on InP
}

\author{
Y.S. Yong $\cdot$ H.Y. Wong $\cdot$ H.K. Yow $\cdot$ M. Sorel
}

Received: 23 May 2009 / Revised version: 20 December 2009 / Published online: 9 April 2010

(C) The Author(s) 2010. This article is published with open access at Springerlink.com

\begin{abstract}
The band-gap energy and the band line-up of InGaAlAs quaternary compound material on InP are essential information for the theoretical study of physical properties and the design of optoelectronics devices operating in the long-wavelength communication window. The bandgap interpolation of $\operatorname{In}_{1-x-y} \mathrm{Ga}_{x} \mathrm{Al}_{y}$ As on InP is known to be a challenging task due to the observed discrepancy of experimental results arising from the bowing effect. Besides, the band line-up results of $\mathrm{In}_{1-x-y} \mathrm{Ga}_{x} \mathrm{Al}_{y} \mathrm{As}$ on InP based on previously reported models have limited success by far. In this work, we propose an interpolation solution using the single-variable surface bowing estimation interpolation method for the fitting of experimentally measured $\mathrm{In}_{1-x-y} \mathrm{Ga}_{x} \mathrm{Al}_{y} \mathrm{As}$ band-gap data with various degree of bowing using the same set of input parameters. The suggested solution provides an easier and more physically interpretable way to determine not only lattice matched, but also strained band-gap energy of $\operatorname{In}_{1-x-y} \mathrm{Ga}_{x} \mathrm{Al}_{y} \mathrm{As}$ on InP based on the experimental results. Interpolated results from this convenient method show a more favourable match to multiple independent experiment data sets measured under different temperature conditions as compared to those obtained from the commonly used weighted-sum approach. On top of that, extended framework of the model-solid theory for the band line-up of $\operatorname{In}_{1-x-y} \mathrm{Ga}_{x} \mathrm{Al}_{y} \mathrm{As} / \mathrm{InP}$ heterostruc-
\end{abstract}

Y.S. Yong $(\bowtie) \cdot$ H.Y. Wong $\cdot$ H.K. Yow

Centre for Advanced Devices and Systems, Faculty

of Engineering, Multimedia University, Jalan Multimedia,

63100 Cyberjaya, Selangor, Malaysia

e-mail: yong.yean.sheng@mmu.edu.my

M. Sorel

Optoelectronics Research Group, Department of Electronics and Electrical Engineering, University of Glasgow, Rankine Building, Oakfield Avenue, Glasgow G12 8LT, UK ture is proposed. Our model-solid theory band line-up result using the proposed extended framework has shown an improved accuracy over those without the extension. In contrast to some previously reported works, it is worth noting that the band line-up result based on our proposed extended model-solid theory has also shown to be more accurate than those given by Harrison's model.

\section{Introduction}

The $\mathrm{In}_{1-x-y} \mathrm{Ga}_{x} \mathrm{Al}_{y}$ As quaternary alloy has drawn great interest in the optoelectronic devices operating in the longwavelength region in the past two decades [1] because of the numerous advantages that it has over its $\operatorname{In}_{1-x} \mathrm{Ga}_{x} \mathrm{As}_{y} \mathrm{P}_{1-y}$ counterpart, such as wider achievable range of refractive index [1], better material gain [2], and higher conduction band discontinuity $[3,4]$. Generally, the band-gap energy and the band line-up of the $\mathrm{In}_{1-x-y} \mathrm{Ga}_{x} \mathrm{Al}_{y}$ As alloy on InP substrate are essential information for the design of these optoelectronic devices. However, despite their importance in the theoretical study of physical properties and the simulation of optoelectronic devices, there is still lack of easily applicable theoretical models for the band-gap energy and the band line-up of the $\mathrm{In}_{1-x-y} \mathrm{Ga}_{x} \mathrm{Al}_{y} \mathrm{As}$ on InP that show close agreement to the experimental results.

The interpolation of the band-gap energy of $\operatorname{In}_{1-x-y^{-}}$ $\mathrm{Ga}_{x} \mathrm{Al}_{y} \mathrm{As}$, which is inherently an important element of certain band line-up model, is a difficult task due to the disagreement of the linearity relationship of the band-gap to the aluminium content [5]. The existence of non-linear dependence in the band-gap energy to the aluminium content, also known as the bowing effect, of $\mathrm{In}_{1-x-y} \mathrm{Ga}_{x} \mathrm{Al}_{y} \mathrm{As}$ has been well supported by the experiment [6-8] and theoretical [9] results. Contrarily, independent measure- 
ment results [10-14] showed linear variations of bandgap energy to the aluminium content. To date, there are a range of interpolation techniques available for the determination of $\mathrm{In}_{1-x-y} \mathrm{Ga}_{x} \mathrm{Al}_{y}$ As band-gap energy. There is the $\mathrm{In}_{0.53} \mathrm{Ga}_{0.47} \mathrm{As}-\mathrm{Al}_{0.48} \mathrm{In}_{0.52} \mathrm{As}$ direct interpolation approach [5], but this approach is limited to lattice matched system and not extendable for strained material systems. Among the interpolation methods capable for the determination of strained band-gap energy, the multivariable technique [15] can only be fitted to one of the above results at a time, whereas the weighted-sum approach [16] requires tedious adjustment of independent ternary bowing parameters to incorporate the bowing effect. Recently, an improved interpolation method with surface bowing estimations was proposed by Mei [17], but there is still lack of a proper mechanism to address the bowing issue of $\mathrm{In}_{1-x-y} \mathrm{Ga}_{x} \mathrm{Al}_{y} \mathrm{As}$ on InP. Considering the disagreement arose on the degree of band-gap bowing in the reported results, multiple sets of interpolation input values will be needed to match the interpolation results to the respective experimental results if these interpolation techniques were to be used. In this paper, an interpolation solution using Mei's single-variable surface bowing estimation interpolation method is proposed. In contrast to the above mentioned interpolation methods, the proposed solution addresses the bowing effect through the adjustment of only a single parameter while leaving the rest of the input parameters as fixed values, which is simple and not only applicable to lattice matched, but also to the strained material systems as well. Other than demonstrating the capability of the proposed solution to address the bowing effect of experimental results via single parameter, a set of material parameters suitable for the band-gap interpolation of $\mathrm{In}_{1-x-y} \mathrm{Ga}_{x} \mathrm{Al}_{y} \mathrm{As}$ on InP will also be determined and presented in this work. Furthermore, the possible physical meaning of the adjustable parameter, which is resultant from mathematical derivation in Mei's work [17], will also be discussed.

On the other hand, simple models are essential for the determination of material system's band line-up for the case of physical behaviour calculation at the device level so that one can focus on the remaining design parameters based on the simple models' result. Standard simple models such as Harrison's model [18] and the model-solid theory [19] had been successfully applied to the ternary alloys. However, there are limited reports on the application of these models to the quaternary alloy which has received tremendous interest in recent years in the design of a spectrum of advanced optoelectronics devices. Band line-up results of the $\mathrm{In}_{1-x-y} \mathrm{Ga}_{x} \mathrm{Al}_{y} \mathrm{As} / \mathrm{InP}$ heterostructure using Harrison's model and the model-solid theory by linearly interpolating the band positions of individual binary compound materials had been reported, but they were both in poor agreement to the empirically determined results [20]. This shows that the linear interpolation approach is inadequate for the band line-up of $\operatorname{In}_{1-x-y} \mathrm{Ga}_{x} \mathrm{Al}_{y} \mathrm{As} / \mathrm{InP}$ heterostructure, and a proper quaternary interpolation framework is needed. In this paper, an extension framework based on the model-solid theory is proposed to cater for the accurate band line-up determination for quaternary alloy.

In Sect. 2, analysis on the available band-gap interpolation techniques, along with their underlying problems are discussed. A solution to the interpolation problems is proposed. In Sect. 3, a review on the model-solid theory is presented. A new proposed extension framework to the modelsolid theory for the determination of band line up is described. In Sect. 4, calculated results of band-gap energy and band line-up based on our proposed interpolation and new framework will be presented. Our results are compared and discussed with existing experimental measurement values and theoretical calculation results for bench marking purposes. Lastly, a conclusion on the work will be made in Sect. 5.

\section{Analysis of the existing band-gap interpolation methods and the new proposed interpolation solution}

The most quoted, and probably the first, report of the bandgap energy of $\mathrm{In}_{1-x-y} \mathrm{Ga}_{x} \mathrm{Al}_{y}$ As (denoted as InGaAlAs hereafter) over a wide composition range lattice matched to InP was published by Olego et al. in 1982. From the photoluminescence (PL) measurement on the samples grown by molecular beam epitaxy (MBE), the empirically determined band-gap energy variation of InGaAlAs to the aluminium content, $y$, was expressed as the analytical formula below [7]:

$E_{\mathrm{g}}=0.76+1.04 y+0.87 y^{2} \mathrm{eV}$,

with the lattice matching condition to InP substrate $x+y \approx$ 0.47 .

The non-linear term in (1) indicates that the band-gap energy of InGaAlAs cannot be interpolated by the simple and convenient Vergard's law which is applicable to most of the other material parameters as follow [21]:

$Q(x, y)=x P_{\mathrm{GaAs}}+y P_{\mathrm{AlAs}}+(1-x-y) P_{\mathrm{InAs}}$,

where $P_{\mathrm{GaAs}}, P_{\mathrm{AlAs}}$, and $P_{\mathrm{InAs}}$ are the material parameters of GaAs, AlAs, and InAs respectively.

In practice, a bowing term was introduced to address the non-linear behaviour when it comes to the interpolation of the band-gap energy of this compound material. This can be achieved by relating the band-gap energies of the two ternary end points lattice matched to InP, represented by the expression of $\left(\mathrm{Al}_{0.48} \mathrm{In}_{0.52} \mathrm{As}\right)_{z}\left(\mathrm{Ga}_{0.47} \mathrm{In}_{0.53} \mathrm{As}\right)_{1-z}$, using the interpolation formula given by [5]:

$$
E_{\mathrm{g}}(z)=(1-z) E_{\mathrm{g}, \mathrm{GaInAs}}+z E_{\mathrm{g}, \mathrm{AlInAs}}-z(1-z) C,
$$


where $E_{\mathrm{g}, \mathrm{GaInAs}}$ and $E_{\mathrm{g} \text {, AlInAs }}$ are the band-gap energies of $\mathrm{Ga}_{0.47} \mathrm{In}_{0.53}$ As and $\mathrm{Al}_{0.48} \operatorname{In}_{0.52}$ As respectively, and $C$ is the bowing parameter.

However, the interpolation result of (3) is only limited to lattice matched layers. In other words, it is not capable of determining the band-gap energy of strained InGaAlAs layers which are widely used in today's optoelectronic devices. Therefore, it will not be the focus of this work. Instead, we would discuss the weighted-sum interpolation scheme, which provides the flexibility of altering the mole fraction of the gallium and aluminium composition independently, thus making it possible to calculate not only the lattice matched but strained material band-gap energy. The weighted-sum approach takes into account the band-gap energies of all ternary constituents, and the band-gap energy of InGaAlAs can be found with the expressions as follow [16]:

$Q(x, y)=\frac{x y T_{A B D}(u)+y z T_{B C D}(v)+x z T_{A C D}(w)}{x y+y z+x z}$,

where

$z \equiv 1-x-y$,

$u \equiv(1-x+y) / 2$,

$v \equiv(1-y+z) / 2$,

$w \equiv(1-x+z) / 2$,

and the $T_{A B D}, T_{B C D}$ and $T_{A C D}$ are the band-gap energies of the ternary alloys which may be interpolated with the expression of:

$T_{P Q R}(x)=(1-x) B_{P R}+x B_{Q R}-x(1-x) C_{P Q R}$,

where $B_{P R}$ and $B_{Q R}$ correspond to the band-gap energies of the related binary constituents and $C_{P Q R}$ is the ternary interpolation bowing parameter.

As mentioned in the previous section, the bowing value is known to be contentious, and most of the bowing values are deduced from (3) but not from the weighted-sum scheme. For instance, fitting of Olego's result [7] using (3) would yield a bowing value of $0.20 \mathrm{eV}$. Other than that, bowing values of $0.22 \mathrm{eV}$ [5], and $0.257 \mathrm{eV}$ [9] had also been suggested by independent reports. A closer look at (4) would reveal that other than changing the distinctive ternary bowing values by smart guesses, there is no direct way to adopt these bowing values into the weighted-sum scheme. Moreover, forcefully fitting of the results of weighted-sum scheme to the experimental measurements with various degree of bowing would only yield different sets of ternary bowing values, resulting in a lack of common basis for the physical interpretation of the interpolation results. Therefore, it is desirable to have a mechanism of interpolation which uses only a single set of parameters, yet the results may be easily fitted to take into account the bowing effect.
Recently, an interpolation method with surface bowing estimations [17] had been developed for the quaternary III-V compound material. With the inclusion of balancing surface bowing estimation error, the band-gap energy of $\mathrm{In}_{1-x-y} \mathrm{Ga}_{x} \mathrm{Al}_{y} \mathrm{As}$ may be calculated with the expression of [21]:

$E_{\mathrm{g}}(x, y, z)=E_{\mathrm{g}, 0}(x, y, z)+\Delta E_{\mathrm{g}}(x, y, z)$,

where $z=1-x-y$, and the $E_{\mathrm{g}, 0}$ is the fundamental estimation, given by

$$
\begin{aligned}
E_{\mathrm{g}, 0}= & x B_{\mathrm{GaAs}}+y B_{\mathrm{AlAs}}+z B_{\mathrm{InAs}}-x y C_{\mathrm{GaAlAs}} \\
& -y z C_{\mathrm{AlInAs}}-x z C_{\mathrm{GaInAs}},
\end{aligned}
$$

with $B_{\mathrm{GaAs}}, B_{\mathrm{AlAs}}$, and $B_{\mathrm{InAs}}$ representing the band-gap energies of GaAs, AlAs, and InAs respectively, and the $C_{\mathrm{GaAlAs}}, C_{\mathrm{AlInAs}}, C_{\mathrm{GaInAs}}$ are the ternary bowing terms similar to that of (5). The $\Delta E_{\mathrm{g}}$ is known as the correction term, which can be determined with the general expression of:

$\Delta E_{\mathrm{g}}=x y z\left(d_{0}+d_{1} x+d_{2} y\right)$,

where $d_{0}, d_{1}$, and $d_{2}$ are the arbitrarily parameters.

In contrast to the weighted-sum approach, the fitting of the band-gap energy using the interpolation method with surface bowing estimations does not depend on the change of material parameters, but it is performed through the adjustment of the adjustable parameters in (6c). Since the binary band-gap energies and ternary bowing values are the basis of any quaternary interpolation method, this inherit nature of the surface bowing interpolation method is deemed to provide a better fitting framework than the weighted-sum method. However, though the surface bowing interpolation method had been validated against the $\mathrm{In}_{0.53} \mathrm{Ga}_{0.47} \mathrm{As}-\mathrm{Al}_{0.48} \mathrm{In}_{0.52} \mathrm{As}$ direct interpolation approach given in (3) [17], there is still no investigation on the applicability and the effectiveness of this method on experimentally measured $\mathrm{In}_{1-x-y} \mathrm{Ga}_{x} \mathrm{Al}_{y}$ As band-gap energy. Consequently, the issue of bowing discrepancy is also left unaddressed. Moreover, with the existence of as much as three arbitrarily adjustable parameters, the fitting process may not be simple and multiple sets of values may arise as the solutions of the fitting work.

To solve the above-mentioned issues, we propose an interpolation solution which may be catered for bowing adjustment and applicable for both the lattice matched and strained band-gap energy of $\operatorname{In}_{1-x-y} \mathrm{Ga}_{x} \mathrm{Al}_{y}$ As on InP. We adopt the single-variable surface bowing interpolation method in which the correction term in (6c) is replaced by a single-variable expression [17].

$\Delta E_{\mathrm{g}}=C_{0} x y z$ 
where the $C_{0}$ is an arbitrary constant in which we will address it as the surface bowing constant hereafter to avoid possible confusion with the $C$ in (3). Notice that the surface bowing constant has no physical meaning in the original work of the interpolation scheme with surface bowing estimations [17]. In this work, attempt will be made to provide a physical meaning to the surface bowing constant. Besides, suitable material parameters used for the band-gap interpolation of InGaAlAs on InP will also be evaluated and presented as part of the solution.

\section{Model-solid theory with the proposed extension framework}

The simulation or theoretical studies of the physical behaviour of optoelectronics devices often require intense calculations on various physical quantities. Therefore, simple model or theory capable of predicting the band offsets for a wide variety of interfaces with adequate accuracy are more preferable than the heavy first-principle calculations based on local density-functional theory and ab initio pseudopotentials in such context. The model-solid theory [19] is based on an absolute reference energy level, and the major distinction of the model-solid theory from other model theories, such as the Harrison's model [18], is that it provides a natural way of dealing with strained-layer interfaces.

Consider the growth of a thin epitaxial layer (i.e. material 2) on another material (i.e. material 1) as shown in Fig. 1. Given that material 1 is a substrate with relatively large layer thickness, $h_{1} \gg h_{2}$, the lattice constant of the epitaxial layer grown on the substrate with (001) orientation in the parallel and perpendicular directions would be:

$a_{\|}=a_{1}$
$a_{\perp}=a_{2}\left[1-2\left(c_{12} / c_{11}\right)\left(a_{1} / a_{2}-1\right)\right]$

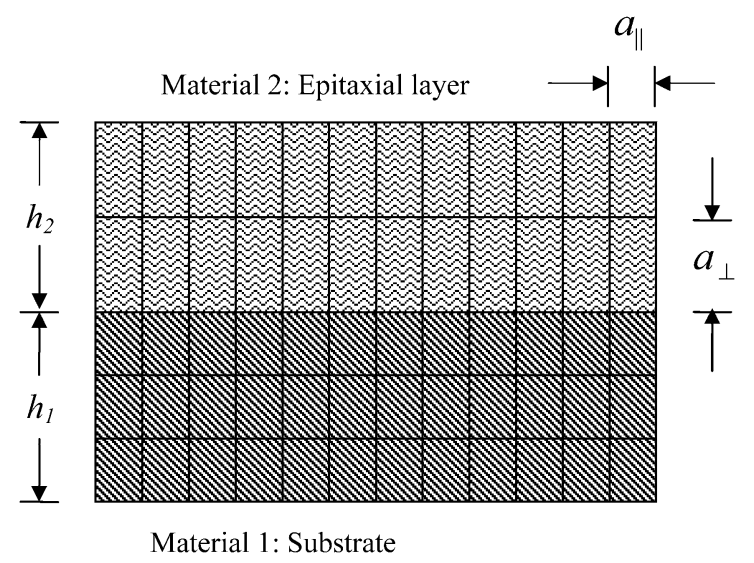

Fig. 1 Reference diagram for the calculation of strain induced energy level shift components respectively, where $a_{1}$ and $a_{2}$ are the equilibrium lattice constants of material 1 and material 2 respectively, and $c_{11}$ and $c_{12}$ are the elastic constants of the epitaxy layer. The resulting strains on the epitaxial layer are given by

$\varepsilon_{\|}=a_{\|} / a_{2}-1$,

$\varepsilon_{\perp}=a_{\perp} / a_{2}-1$.

Without the influence of shear component induced effect, the position of the topmost valence band is determined by the average valence band energy level, $E_{\mathrm{v}, \text { av }}$ and the shift due to the hydrostatic deformation potential with the expression of:

$$
\begin{aligned}
E_{\mathrm{v}} & =E_{\mathrm{v}, \mathrm{av}}+\frac{\Delta_{\mathrm{so}}}{3}+a_{\mathrm{v}}\left(2 \varepsilon_{\|}+\varepsilon_{\perp}\right) \\
& =E_{\mathrm{v}, \mathrm{av}}+\frac{\Delta_{\mathrm{so}}}{3}+2 a_{\mathrm{v}}\left(1-\frac{c_{12}}{c_{11}}\right) \varepsilon_{\|},
\end{aligned}
$$

where $\Delta_{\text {so }}$ is the spin-orbit splitting constant, and $a_{\mathrm{v}}$ is the hydrostatic deformation potential for the valence band.

Since the band-gap energy of lattice matched InGaAlAs falls within the direct band-gap region, the conduction band is only subjected to the hydrostatic strain shift:

$$
\begin{aligned}
E_{\mathrm{c}} & =E_{\mathrm{v}}+E_{\mathrm{g}}+a_{\mathrm{c}}\left(2 \varepsilon_{\|}+\varepsilon_{\perp}\right) \\
& =E_{\mathrm{v}}+E_{\mathrm{g}}+2 a_{\mathrm{c}}\left(1-\frac{c_{12}}{c_{11}}\right) \varepsilon_{\|},
\end{aligned}
$$

where $a_{\mathrm{c}}$ is the hydrostatic deformation potential for the conduction band, and $E_{\mathrm{g}}$ is the band-gap energy.

On the other hand, additional splitting due to the shear component of the strain has to be taken into account when determining the energy level for the heavy hole, light hole, and split-off bands, resulting in the band position expressions of:

$$
\begin{aligned}
& E_{\mathrm{hh}}=E_{\mathrm{v}}-\frac{\delta E}{2}, \\
& E_{\mathrm{lh}}=E_{\mathrm{v}}-\frac{\Delta_{0}}{2}+\frac{\delta E}{4}+\frac{1}{2}\left[\Delta_{0}^{2}+\Delta_{0} \delta E+\frac{9}{4}(\delta E)^{2}\right]^{1 / 2}, \\
& E_{\mathrm{so}}=E_{\mathrm{v}}-\frac{\Delta_{0}}{2}+\frac{\delta E}{4}-\frac{1}{2}\left[\Delta_{0}^{2}+\Delta_{0} \delta E+\frac{9}{4}(\delta E)^{2}\right]^{1 / 2},
\end{aligned}
$$

and the $\delta E$ is given by

$\delta E=2 b\left(\varepsilon_{\perp}-\varepsilon_{\|}\right)$,

where $b$ is the shear deformation potential.

The values of the parameters mentioned above for the related binary compound materials are listed in Table 1 . 

band line-up calculation of $\mathrm{In}_{1-x-y} \mathrm{Ga}_{x} \mathrm{Al}_{y} \mathrm{As}$ quaternary alloy on InP substrate [20]
Table 1 Parameters for the

\begin{tabular}{lllllllll}
\hline & $\begin{array}{l}a \\
(\AA)\end{array}$ & $\begin{array}{l}c_{11} \\
\left(10^{11} \mathrm{dyn} / \mathrm{cm}^{2}\right)\end{array}$ & $\begin{array}{l}c_{12} \\
\left(10^{11} \mathrm{dyn} / \mathrm{cm}^{2}\right)\end{array}$ & $\begin{array}{l}\Delta_{\text {so }} \\
(\mathrm{eV})\end{array}$ & $\begin{array}{l}E_{\mathrm{V}, \mathrm{av}} \\
(\mathrm{eV})\end{array}$ & $\begin{array}{l}a_{\mathrm{v}} \\
(\mathrm{eV})\end{array}$ & $\begin{array}{l}a_{\mathrm{c}} \\
(\mathrm{eV})\end{array}$ & $\begin{array}{l}b \\
(\mathrm{eV})\end{array}$ \\
\hline InP & 5.8688 & 10.110 & 5.610 & 0.11 & -7.04 & 1.27 & -5.04 & -1.7 \\
GaAs & 5.6533 & 11.879 & 5.376 & 0.34 & -6.92 & 1.16 & -7.17 & -1.7 \\
AlAs & 5.6600 & 12.500 & 5.340 & 0.28 & -7.49 & 2.47 & -5.64 & -1.5 \\
InAs & 6.0584 & 8.329 & 4.526 & 0.38 & -6.67 & 1.00 & -5.08 & -1.8 \\
\hline
\end{tabular}

iv. The quaternary value of the other parameters, such as $c_{11}, c_{12}, \Delta_{\mathrm{so}}, a_{\mathrm{c}}, a_{\mathrm{v}}$, and $b$ may be interpolated by Vergard's law.

v. The energy band levels are then calculated by following the ordinary band line-up procedure with (7)-(12) using either the experimental band-gap energy, or the interpolated band-gap energy.

With our proposed framework, the band line-up result will reduce to the band line-up of the respective ternary alloy if one of the $x, y$, or $z$ composition is set to zero. Therefore, previously established agreement of the model-solid theory's results to the ternary alloys can be assumed, and only assessment of the band line-up results at the middle composition range is needed to verify the validity of the proposed framework.

\section{Results}

In order to have a more comprehensive analysis which examines the accuracy of the interpolated results at various temperature, the temperature-dependent band-gap energy of the binary compound materials in (6) are calculated using the Varshni's empirical formula given by [23]:

$E_{\mathrm{g}}(T)=E_{\mathrm{g}}(T=0)-\frac{\alpha T^{2}}{T+\beta}$

coefficients of the corresponding energy level, and the $\Delta a_{0}$ and $a_{0}$ are the lattice mismatch value, and the lattice constant linearly interpolated with Vergard's law respectively.

In order to take advantage of the known accuracy of the band line-up of $\mathrm{In}_{0.53} \mathrm{Ga}_{0.47} \mathrm{As} / \mathrm{InP}$ and $\mathrm{Al}_{0.48} \mathrm{In}_{0.52} \mathrm{As} /$ $\mathrm{In}_{0.53} \mathrm{Ga}_{0.47}$ As heterostructures using model-solid theory, we propose the following quaternary framework, which takes into account the lattice mismatch term according to the scheme outlined in the following main sequential steps:

i. Determination of parameter $a_{0}$ for the $\mathrm{Ga}_{1-u} \mathrm{Al}_{u} \mathrm{As}$, $\mathrm{Al}_{1-v} \mathrm{In}_{v} \mathrm{As}$, and $\mathrm{Ga}_{1-w} \mathrm{In}_{w}$ As using the Vergard's law.

ii. Calculation of $E_{\mathrm{v}, \text { av }}$ for the $\mathrm{Ga}_{1-u} \mathrm{Al}_{u} \mathrm{As}, \mathrm{Al}_{1-v} \mathrm{In}_{v} \mathrm{As}$, and $\mathrm{Ga}_{1-w} \operatorname{In}_{w}$ As separately using (13), where $a_{\mathrm{i}}$ in this case is the hydrostatic deformation potential for the valence band.

iii. Obtain the quaternary $E_{\mathrm{v}, \mathrm{av}}$ by relating the ternary values using the weighted-sum approach. where $\alpha$ and $\beta$ are arbitrary constants, and $T$ is the temperature in Kelvin.

In this work, most of the values of the band-gap parameters are extracted from [5]. However, the set of parameters in [5] is not capable to describe the temperature-dependent band-gap energy of $\operatorname{In}_{0.53} \mathrm{Ga}_{0.47}$ As within acceptable range due to the wide range of difference among the reported values. Therefore, it is important to obtain a suitable set of parameters prior to the demonstration of the proposed interpolation solution. To illustrate the reasoning behind this, the temperature-dependent band-gap energy of $\mathrm{In}_{0.53} \mathrm{Ga}_{0.47} \mathrm{As}$ interpolated from the single-variable surface bowing estimation interpolation approach at default state (i.e. $C_{0}=0$ ) is shown in Fig. 2. The dotted curve in Fig. 2 represents the experimental results of those with higher energy values, and it is generated using the values recommended by [5]. On 


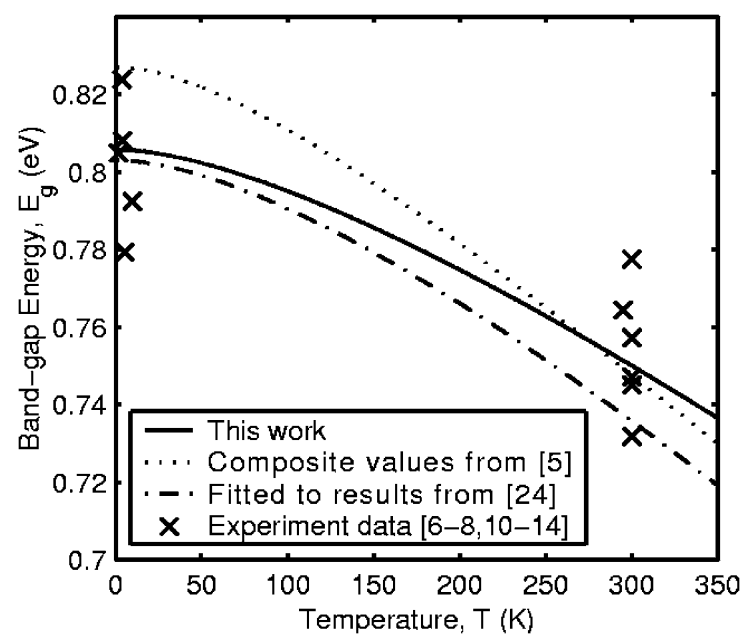

Fig. 2 Fitted band-gap energy of $\mathrm{In}_{0.53} \mathrm{Ga}_{0.47} \mathrm{As}$ from the single-variable interpolation approach at default state, together with the comparison of experimental data from various sources

the other hand, the dash-dot curve represents the results of those with lower energy values, and it is obtained by fitting the interpolation curve to those of Gaskill et al. [24]. The experimental band-gap values in the same figure was obtained from the reports studying the composition-dependent band-gap energy of InGaAlAs [6-8, 10-14]. As observed in Fig. 2, due to the wide spread of experimental band-gap values at near $0 \mathrm{~K}$ and also $300 \mathrm{~K}$, it is difficult to find a single curve that matches all of the experimental data. The dotted curve is near the median of the data at $300 \mathrm{~K}$, whereas the dash-dot curve shows a better match to the data near $0 \mathrm{~K}$. Therefore, in this work, we had fitted the results in such a way that it passes near the median of the data at both near 0 and $300 \mathrm{~K}$, and the result is shown as the solid curve. The solid curve is within the experimental error of the PL measurement on zero mismatch InGaAs grown on semi-insulating InP substrates at $7 \mathrm{~K}$ [25], and the $300 \mathrm{~K}$ band-gap energy was fitted to the widely accepted value of $0.75 \mathrm{eV}[26,27]$. The values of all fitting parameters used are listed in Table 2 for the ease of reference. The solidline result from Fig. 2 together with the other commonly accepted values from [5] are used for the subsequent parts of the manuscript.

Figure 3 shows the interpolated band-gap energy of InGaAlAs lattice matched to InP at $300 \mathrm{~K}$. The interpolation result using weighted-sum approach is also plotted in Fig. 3 for comparison. As shown in Fig. 3, both interpolation results have the same end points which correspond to the ternary band-gap values of $\operatorname{In}_{0.53} \mathrm{Ga}_{0.47} \mathrm{As}$ and $\mathrm{Al}_{0.48} \mathrm{In}_{0.52} \mathrm{As}$, but the weighted-sum result overestimated the band-gap value at the middle of the aluminiumcontent range. On the other hand, the interpolated curve using single-variable surface bowing estimation interpolation approach has a slight s-shape when the correction term is
Table 2 Fitted values for the band-gap parameters of InGaAs alloy lattice matched to InP shown in Fig. 2

\begin{tabular}{lllll}
\hline & $\begin{array}{l}E_{\mathrm{g}, \mathrm{GaAs}}^{\Gamma} \\
(\mathrm{eV})\end{array}$ & $\begin{array}{l}\alpha_{\mathrm{GaAs}}^{\Gamma} \\
(\mathrm{meV} / \mathrm{K})\end{array}$ & $\begin{array}{l}\beta_{\mathrm{GaAs}}^{\Gamma} \\
(\mathrm{K})\end{array}$ & $C_{\mathrm{GaInAs}}$ \\
\hline Dotted line $^{\mathrm{a}}$ & 1.519 & 0.5405 & 204 & 0.4770 \\
Dash-dot line $^{\mathrm{b}}$ & $1.420^{\mathrm{c}}$ & 0.6755 & 550 & 0.3824 \\
Solid line-This work $^{1}$ & $1.420^{\mathrm{c}}$ & 0.5120 & 670 & 0.3715 \\
\hline
\end{tabular}

${ }^{\mathrm{a}}$ From [5]

${ }^{\mathrm{b}}$ Fitted to [24]

${ }^{\mathrm{c}}$ From [31]

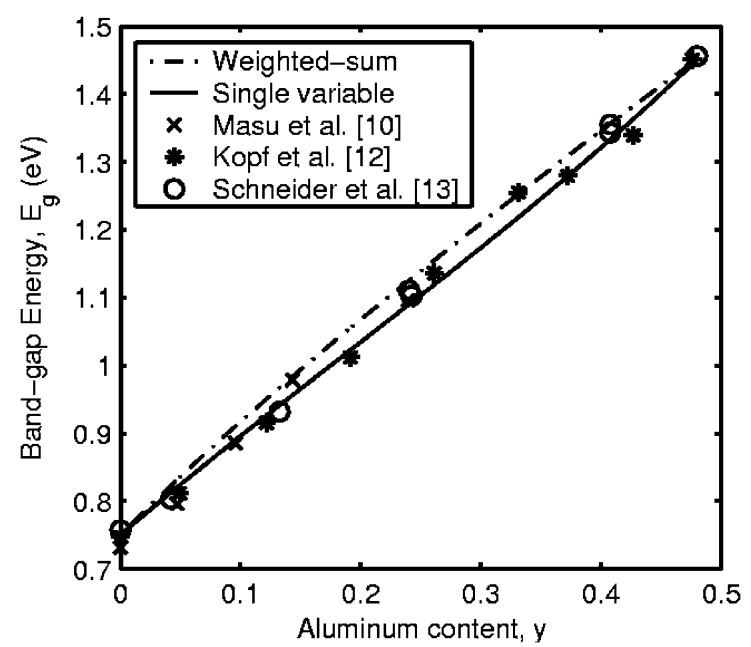

Fig. 3 Interpolation results of $\operatorname{In}_{1-x-y} \mathrm{Ga}_{x} \mathrm{Al}_{y}$ As lattice matched to InP from two interpolation schemes, calculated using the same set of input values, at $300 \mathrm{~K}$. The solid line represents the result of single-variable interpolation approach at default state, whereas the results by weighted-sum approach is shown in dash-dot curve

neglected (i.e. $C_{0}=0$ ), and the results fall in between of the experimental measurement values reported in $[10,12,13]$. As an additional step to verify the band-gap energy at the middle of the aluminium-content range, the interpolation results at $y=0.30$ to the experimental measured result [28] of $1.18 \mathrm{eV}$ are compared. Our calculation from single-variable interpolation approach gives a value of $1.174 \mathrm{eV}$, as compared to the weighted-sum's value of $1.209 \mathrm{eV}$, is closer to the measurement result at $y=0.30$.

To further validate the performance of the single-variable interpolation approach, the result is compared to the experimental band-gap energies at low temperatures, as shown in Fig. 4. The interpolation result is in good agreement with the experimental data measured at $4.2 \mathrm{~K}$ [13], $5.5 \mathrm{~K}$ [12], and $10 \mathrm{~K}$ [11] respectively. The interpolated values matched well and exhibit a maximum deviation of $0.027 \mathrm{eV}$ from the experimental data.

In order to address the bowing effect of the band-gap energy, the surface bowing term in (6c) may be facilitated to 


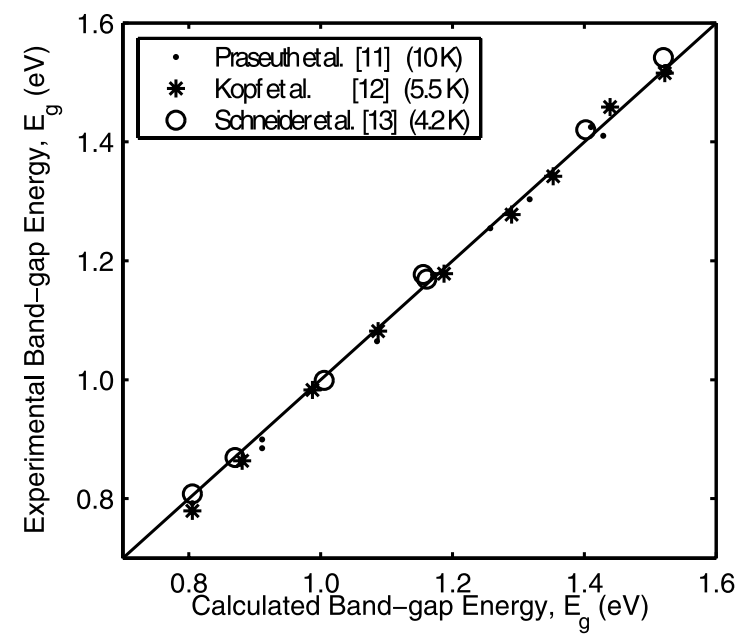

Fig. 4 Comparison of the InGaAlAs band-gap energy values calculated from single-variable interpolation approach at default state in this work to the experimental values under various measurement temperatures

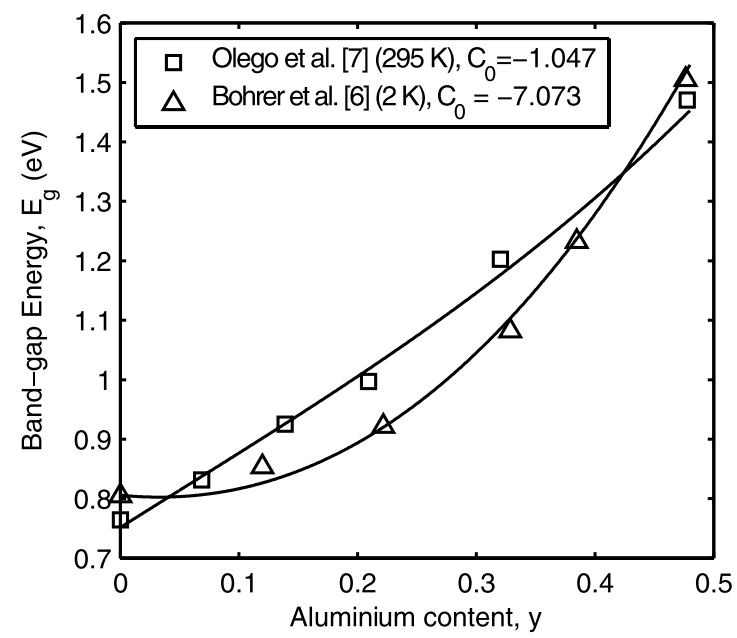

Fig. 5 Interpolation results fitted to experimental data with non-linear dependence of band-gap energy to aluminium content by adjusting the surface bowing constant, $C_{0}$ only

fit the experimental data. The interpolation results best fitted to the experimental data, together with the respective surface bowing value $C_{0}$ determined from the standard leastsquare fitting approach are given in Fig. 5. Note that the two end points of the interpolated results bow to higher energy vales and the band-gap energy at the middle range is reduced when the bowing effect is more significant. The interpolation scheme is not only capable of producing a result fitted well to the commonly accepted Olego's result [7], but also to Böhrer's result [6] with even greater bowing effect. This indicates that it is possible to fit the interpolated bandgap energy to the results with different degree of bowing by changing the $C_{0}$ alone. To fit the same experimental results using weighted-sum approach would need the change of three ternary bowing values, thus complicate the process. The same applies to the surface bowing estimation interpolation method with three arbitrary variable [i.e. (6c)]. Furthermore, with the close agreement of the interpolated lattice matched band-gap energy under different bowing conditions, it is possible to extrapolate the strained band-gap energy based on our proposed solution. One would only need to alter the surface bowing constant to fit the interpolated result to the concerned experimental results. Such a fitting approach is similar to the adjustment of bowing parameter in the $\operatorname{In}_{0.53} \mathrm{Ga}_{0.47} \mathrm{As}-\mathrm{Al}_{0.48} \mathrm{In}_{0.52}$ As direct interpolation approach [i.e. (3)]. With the use of consistent set of material values, the results would be maintained rational even with the use of different bowing values. It will be valuable for the input of device's simulation study as different researchers may choose to adhere to different experimental results.

Both Böhrer et al. [6] and Olego et al. [7] had explained that the non-linearity behaviour in Fig. 5 was probably due to alloy disorder. Assuming that this explanation is valid, it may be justified that $C_{0}$, an arbitrary constant which has no definite physical meaning in the original work of the interpolation scheme with surface bowing estimations [17], is dependent on the degree of alloy disorder, and a higher magnitude of $C_{0}$ value indicates a more severe alloy disorder effect. Similarly, a zero $C_{0}$ value can be interpreted as InGaAlAs data sets with minimal alloy disorder, thus giving a band-gap energy linearly dependent on the aluminium composition.

Generally, an accurate interpolation work should produce results with small mean of deviation, $\bar{\Delta}$ and small maximum of deviation, $\Delta_{\max }$ from the experimental data set. Of all the measurement results presented in Figs. 3 and 4, the interpolation results with single-variable interpolation approach have smaller $\bar{\Delta}$ and $\Delta_{\max }$ than the weighted-sum approach. For some cases, our interpolation results are even better than the relatively simpler analytical expressions. Listed in Table 3 is the selected comparison of $\bar{\Delta}$ and $\Delta_{\max }$ between the weighted-sum interpolation, single-variable interpolation approach, and analytical formula. It is shown, from the comparison of $\bar{\Delta}$ and $\Delta_{\max }$ in Table 3, that our interpolation results are at least on par to, if not better than, that of the analytical formulas.

With regard to the evaluation of $\operatorname{In}_{1-x-y} \mathrm{Ga}_{x} \mathrm{Al}_{y} \mathrm{As} / \mathrm{InP}$ heterostructure interface band line-up, Minch et al. had shown that the Harrison's model matches closer to the empirical results than the model-solid theory [20]. Although Minch's comparison graph contains a minor flaw, observation from the corrected graph indicates that the conclusion made by Minch et al. still hold true [29]. Figure 6 depicts the calculations results adopted from [20] which are compared against the empirical formula [6]. On the other hand, our calculation result, using the same input values in [20], based 
Table 3 Comparison of mean deviation, $\bar{\Delta}$ and maximum deviation, $\Delta_{\max }$ of the interpolated and analytical formulas results from the experimental values

\begin{tabular}{|c|c|c|c|c|c|c|}
\hline & \multicolumn{3}{|c|}{ Mean deviation, $\bar{\Delta}(\mathrm{meV})$} & \multicolumn{3}{|c|}{ Max deviation, $\Delta_{\max }(\mathrm{meV})$} \\
\hline & Weighted-sum & Single variable & Analytical formula & Weighted-sum & Single variable & Analytical formula \\
\hline Kopf et al. ${ }^{\mathrm{a}}(300 \mathrm{~K})$ & 22.49 & 13.50 & 14.18 & 42.64 & 37.23 & 32.36 \\
\hline Praseuth et al. ${ }^{\mathrm{b}}(10 \mathrm{~K})$ & 30.70 & 12.97 & 27.48 & 53.36 & 26.54 & 53.98 \\
\hline Böhrer et al. ${ }^{\mathrm{c}}(2 \mathrm{~K})$ & - & 11.30 & 24.18 & - & 27.12 & 59.62 \\
\hline
\end{tabular}

${ }^{\mathrm{a}}$ From [12]: $E_{\mathrm{g}}=0.752+1.453 y$

${ }^{\mathrm{b}}$ From $[11,32]: E_{\mathrm{g}}=0.80+1.58 y$

${ }^{\mathrm{c}}$ From [6]: $E_{\mathrm{g}}=0.81+0.036 y+2.96 y^{2}$

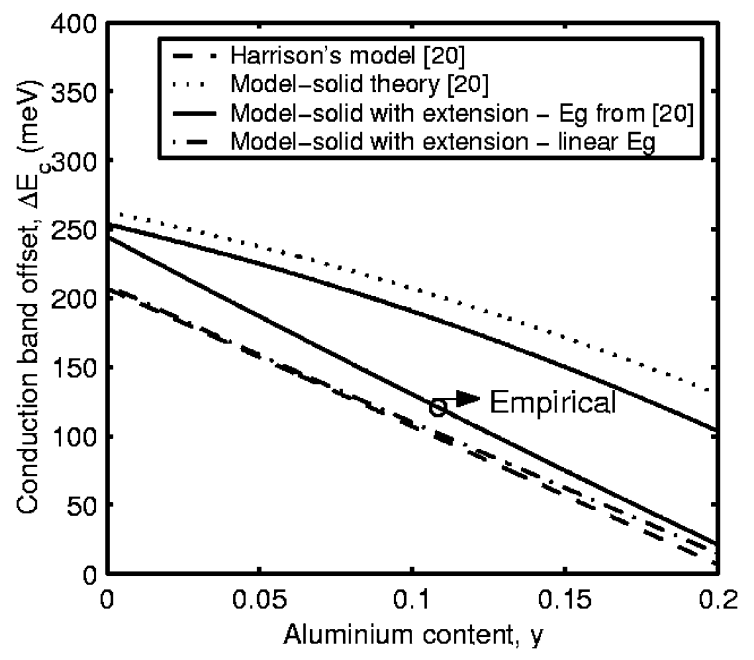

Fig. 6 Comparison of the calculated conduction band offsets for $\mathrm{In}_{1-x-y} \mathrm{Ga}_{x} \mathrm{Al}_{y} \mathrm{As}$ lattice matched to InP to the empirical result in [6]. The dash and dotted line are adjusted according to the calculation in [20], whereas the solid and dash-dot lines are calculated from model-solid theory with quaternary extension framework

on the proposed extension framework is shown in Fig. 6 with a solid line. It is evident that the model-solid result in this work exhibits an improved accuracy over the previous model-solid result in [20]. Since the band-gap energy is an important element in determining the accuracy of the band line-up result in model-solid theory, attempts had been made to fit the result using other sets of band-gap energy. The dash-dot line in Fig. 6 records the best result of the modelsolid theory with extension framework calculated using the linear band-gap result from our single-variable interpolation approach given in Fig. 4. Due to the close proximity of the dash-dot line to the result from Harrison's model, a more detailed comparison is given in Fig. 7, whereby the deviations of the calculated conduction band offset values to the empirical formula [6] are presented. As observed in Fig. 7, result derived from our extended framework has shown smaller deviation to the empirical result than those predicted from the Harrison's model, and its deviation from the empirical re-

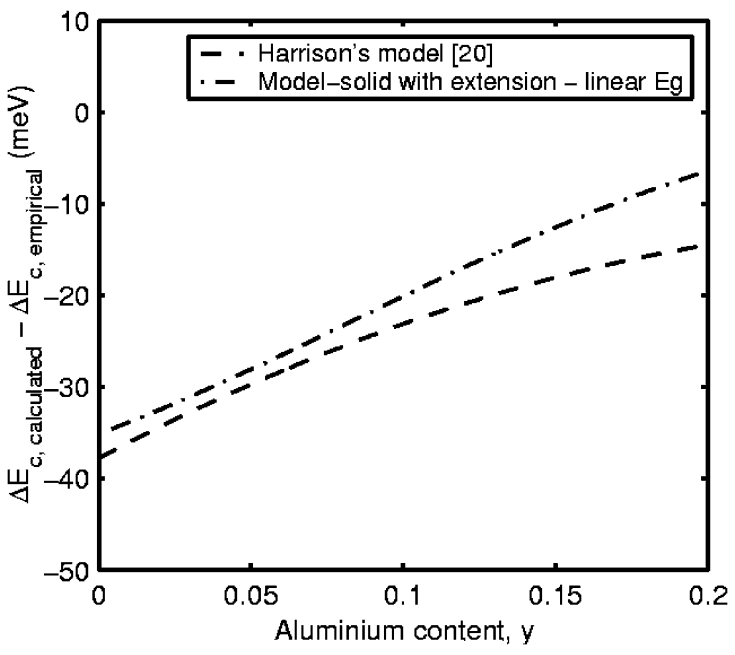

Fig. 7 The difference of the calculated conduction band offsets from the empirical result in [6]. The dash line represents the results of Harrison's model according to [20], and the dash-dot line represents the best result of model-solid theory with quaternary extension framework

sult is always less than $35 \mathrm{meV}$. The comparison of the three model-solid results in Fig. 6 demonstrates that the inclusion of the proposed extension framework, together with a proper choice of band-gap energy may substantially improve the accuracy of the conduction band offset energy produced by model-solid theory.

Since there is a lack of experimental band line-up result of $\operatorname{In}_{1-x-y} \mathrm{Ga}_{x} \mathrm{Al}_{y}$ As on InP over a wide composition range, we further verify the findings by comparing InGaAlAs $/ \mathrm{Al}_{0.48} \mathrm{In}_{0.52} \mathrm{As}$ band line-up results to the theoretical calculation results using the first-principles pseudopotential method under virtual crystal approximation [30], as shown in Fig. 8. The solid line in Fig. 8 is calculated using the model-solid theory with the proposed extension framework, and the results from the Harrison's model and the model-solid theory in [20] are given in dash lines and dotted line, respectively for comparison purpose. Figure 8 shows that among the three band line-up results, the band offsets 


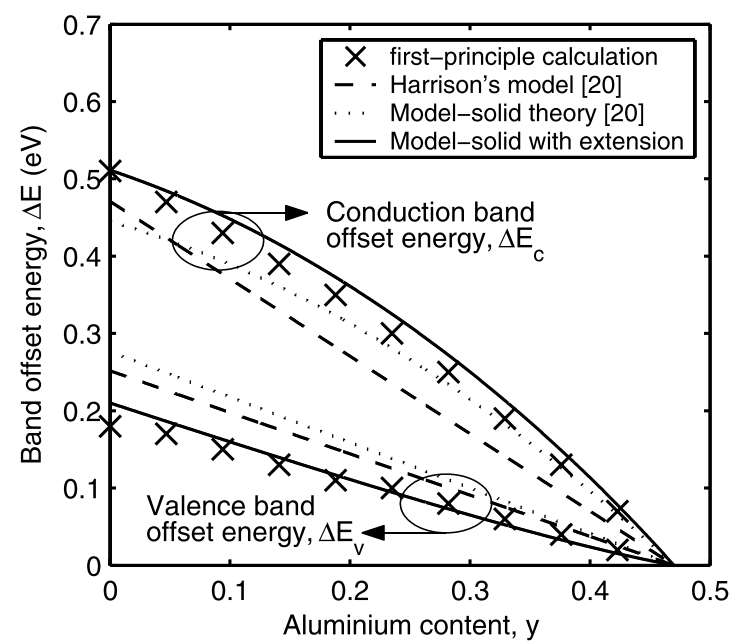

Fig. 8 Conduction band offset $\Delta E_{\mathrm{c}}$ and valence band offset $\Delta E_{\mathrm{v}}$ energies for $\mathrm{In}_{1-x-y} \mathrm{Ga}_{x} \mathrm{Al}_{y} \mathrm{As} / \mathrm{InAlAs}$ interface lattice matched to InP

produced from model-solid theory with extension framework give the most accurate band offsets description with respect to the first-principle result. It is worthwhile to note that our results from model-solid theory with quaternary extension framework lies within the $0.03 \mathrm{eV}$ error bar of the first-principle calculation for the entire range of aluminium composition, which is remarkably good since the model-solid theory was expected to give results typically within $\pm 0.2 \mathrm{eV}$ to the values obtained from the typical self-consistent calculations [19]. The good agreement of the band offset values produced indicates that the effectiveness of the proposed framework in extending the model-solid theory to describe the band line-up of InGaAlAs/InP heterostructure interface. Moreover, in contrast to the argument made in [20], we have demonstrated, in Figs. 6-8, that our extended framework can well be more accurate than the Harrison's model.

\section{Conclusion}

An InGaAlAs band-gap interpolation approach capable of giving a good band-gap result under different temperature and bowing conditions is proposed. The interpolation results are verified against various sets of experiment results and agree well with the experimental values measured at the temperatures of $2,4.2,5.5,10,295$, and $300 \mathrm{~K}$. The proposed approach is better than the more conventional weighted-sum approach in terms of accuracy and ease of fitting to experimental results with significant alloy disorder by adjusting a single variable, while maintaining the same set of the material input values. In addition, a new interpolation framework based on model-solid theory for the band line-up of $\mathrm{In}_{1-x-y} \mathrm{Ga}_{x} \mathrm{Al}_{y} \mathrm{As}$ on InP is suggested. The results of band line-up calculation using this simple extended framework are shown to be in good agreement with the result from first-principle calculation as well as shown to be more accurate in comparison to the model-solid theory without extended framework and also the Harrison's model. The band-gap interpolation and the band line-up methods proposed in this work would be useful to provide simple and accurate means for any future design and study of optoelectronic devices based on $\mathrm{In}_{1-x-y} \mathrm{Ga}_{x} \mathrm{Al}_{y}$ As quaternary systems.

Acknowledgement This work was supported by the Malaysian Ministry of Science, Technology, and Innovation (MOSTI) under EScience Grant 03-02-01-SF0030.

Open Access This article is distributed under the terms of the Creative Commons Attribution Noncommercial License which permits any noncommercial use, distribution, and reproduction in any medium, provided the original author(s) and source are credited.

\section{References}

1. M. Allovon, M. Quillec, Proc. Inst. Electr. Eng. 139, 148 (1992)

2. J.C.L. Yong, J.M. Rorison, R.V. Penty, I.H. White, in Tech. Dig. CLEO (2001), p. 479

3. R. People, K.W. Wecht, K. Alavi, A.Y. Cho, Appl. Phys. Lett. 43, 118 (1983)

4. S.R. Forrest, P.H. Schmidt, R.B. Wilson, M.L. Kaplan, Appl. Phys. Lett. 45, 1199 (1984)

5. I. Vurgaftman, J.R. Meyer, L.R. Ram-Mohan, J. Appl. Phys. 89, $5815(2001)$

6. J. Böhrer, A. Krost, D.B. Bimberg, Appl. Phys. Lett. 63, 1918 (1993)

7. D. Olego, T.Y. Chang, E. Silberg, E.A. Caridi, A. Pinczuk, Appl. Phys. Lett. 41, 476 (1982)

8. P. Parayanthal, C.S. Ro, F.H. Pollak, C.R. Stanley, G.W. Wicks, L.F. Eastman, Appl. Phys. Lett. 43, 109 (1983)

9. M. Marques, L.K. Teles, L.G. Ferreira, L.M.R. Scolfaro, J. Furthmuller, F. Bechstedt, Phys. Rev. B 73, 235205 (2006)

10. K. Masu, T. Mishima, S.-i. Hiroi, M. Konagai, K. Takahashi, J. Appl. Phys. 53, 7558 (1982)

11. J.P. Praseuth, M.C. Joncour, J.M. Gerard, P. Henoc, M. Quillec, J. Appl. Phys. 63, 400 (1988)

12. R.F. Kopf, H.P. Wei, A.P. Perley, G. Livescu, Appl. Phys. Lett. 60, 2386 (1992)

13. J.M. Schneider, J.T. Pietralla, H. Heinecke, J. Cryst. Growth 175176, 184 (1997)

14. J.I. Davies, A.C. Marshall, M.D. Scott, R.J.M. Griffiths, Appl. Phys. Lett. 53, 276 (1988)

15. G.P. Donati, R. Kaspi, K.J. Malloy, J. Appl. Phys. 94, 5814 (2003)

16. C. Williams, T. Glisson, J. Hauser, M. Littlejohn, J. Electron. Mater. 7, 639 (1978)

17. T. Mei, J. Appl. Phys. 101, 013520 (2007)

18. W.A. Harrison, J. Vac. Sci. Technol. 14, 1016 (1977)

19. C.G. Van de Walle, Phys. Rev. B 39, 1871 (1989)

20. J. Minch, S.H. Park, T. Keating, S.L. Chuang, IEEE J. Quantum Electron. 35, 771 (1999)

21. S.L. Chuang, Physics of Optoelectronic Devices (WileyInterscience, New York, 1995)

22. M. Cardona, N.E. Christensen, Phys. Rev. B 37, 1011 (1988)

23. Y.P. Varshni, Physica 34, 149 (1967)

24. D.K. Gaskill, N. Bottka, L. Aina, M. Mattingly, Appl. Phys. Lett. 56, 1269 (1990) 
25. C. Bassignana, C.J. Miner, N. Puetz, J. Appl. Phys. 65, 4299 (1989)

26. Y. Takeda, A. Sasaki, Y. Imamura, T. Takagi, J. Appl. Phys. 47, 5405 (1976)

27. T. Pearsall, IEEE J. Quantum Electron. 16, 709 (1980)

28. R. Rinaldi, R. Cingolani, M. Ferrara, L. Tapfer, H. Kunzel, A. Hase, J. Appl. Phys. 73, 898 (1993)
29. Y.-S. Yong, H.-Y. Wong, H.-K. Yow, IEEE J. Quantum Electron. 45, 1302 (2009)

30. X.H. Zhang, S.J. Chua, S.J. Xu, W.J. Fan, J. Appl. Phys. 83, 5852 (1998)

31. S. Adachi, J. Appl. Phys. 53, 8775 (1982)

32. W.J. Keeler, G.A. Keeler, D.A. Harrison, Z.R. Wasilewski, J. Appl. Phys. 83, 2266 (1998) 